\title{
SUPERNILPOTENCE PREVENTS DUALIZABILITY
}

\author{
WOLFRAM BENTZ ${ }^{\bowtie}$ and PETER MAYR
}

(Received 27 November 2012; accepted 8 August 2013; first published online 30 September 2013)

\author{
Communicated by M. Jackson
}

\begin{abstract}
We address the question of the dualizability of nilpotent Mal'cev algebras, showing that nilpotent finite Mal'cev algebras with a nonabelian supernilpotent congruence are inherently nondualizable. In particular, finite nilpotent nonabelian Mal'cev algebras of finite type are nondualizable if they are direct products of algebras of prime power order. We show that these results cannot be generalized to nilpotent algebras by giving an example of a group expansion of infinite type that is nilpotent and nonabelian, but dualizable. To our knowledge this is the first construction of a nonabelian nilpotent dualizable algebra. It has the curious property that all its nonabelian finitary reducts with group operation are nondualizable. We were able to prove dualizability by utilizing a new clone theoretic approach developed by Davey, Pitkethly, and Willard. Our results suggest that supernilpotence plays an important role in characterizing dualizability among Mal'cev algebras.
\end{abstract}

2010 Mathematics subject classification: primary 03C05; secondary 08A05, 08B05, 08C15.

Keywords and phrases: natural duality, Mal'cev algebra, nilpotence, partial clones.

\section{Introduction}

Natural dualities are representations of elements of an algebra as continuous structure preserving maps (obtained in a certain natural way). For example, Stone duality represents a Boolean algebra as the algebra of characteristic functions of the clopen subsets of a Boolean space. A finite algebra $\mathbf{A}$ is dualizable if every algebra from the quasivariety generated by $\mathbf{A}$ has such a representation.

The first author has received funding from the European Union Seventh Framework Programme (FP7/2007-2013) under grant agreement no. PCOFUND-GA-2009-246542 and from the Foundation for Science and Technology of Portugal. The second author acknowledges support from the Portuguese Project PEst-OE/MAT/UI0143/2011 of CAUL financed by FCT and FEDER and from grant P24285 of the Austrian Science Fund FWF.

(C) 2013 Australian Mathematical Publishing Association Inc. 1446-7887/2013 \$16.00 
Clark and Davey [4, page 291] posed the challenge 'Characterize the dualizable finite algebras in a given class C of algebras', suggesting (among other options) letting $\mathbf{C}$ be the class of all algebras generating a congruence permutable variety (that is, algebras with a Mal'cev term). Recently at the Conference on Order, Algebra, and Logics in Nashville 2007, Willard [17] revived this question in light of a new approach to showing dualizability (see Corollary 4.3).

While Mal'cev algebras are in general considered to be well understood, the question of their dualizability has so far only been addressed for various classes of classical algebras (see below for examples). This contrasts sharply with the situation for algebras in congruence distributive varieties, which have been shown to be dualizable if and only if they have a near-unanimity term [7].

In this paper, we will make a start on the dualizability problem for Mal'cev algebras by examining the role of nilpotence. As usual, we will restrict commutator theoretic properties to the setting of congruence modular varieties; note that every nilpotent congruence modular algebra is a Mal'cev algebra [9, Theorem 6.2].

Our interest in nilpotence comes from the observation that in the previously classified classes of Mal'cev algebras, all dualizable nilpotent algebras were in fact abelian. As we will see, this property is false for Mal'cev algebras in general, but holds if one replaces nilpotence with the slightly stronger condition of supernilpotence. Without directly referring to supernilpotence, our main theorem can be expressed as follows.

THEOREM 1.1. Let $\mathbf{A}$ be a finite nilpotent algebra of finite type in a congruence modular variety. Assume that $\mathbf{A}$ is nonabelian and a direct product of algebras of prime power order. Then $\mathbf{A}$ is inherently nondualizable.

This implies the nondualizability part of several known results, for example:

(1) a finite group is dualizable if and only if its Sylow subgroups are abelian [13, 14];

(2) a finite commutative ring with 1 is dualizable if and only if the square of its Jacobson radical is 0 [6];

(3) a finite ring (without 1) is not dualizable if it contains a nilpotent subring that is not a zero-ring [15].

The finite nonabelian loops whose multiplication group (the group generated by all left and right translations) is nilpotent are an application of the theorem to a class of algebras that were not previously known to be nondualizable ([16, Proposition 3.2], see also [2, Corollary III, page 282]).

We will prove Theorem 1.1 in Section 3. In fact, there we will obtain a more general nondualizability result for nilpotent algebras with a nonabelian, supernilpotent congruence (Theorem 3.1). In the next section we will define this notion of supernilpotence (a stronger condition than nilpotence), give equivalent formulations of the conditions of the theorem, and prove several auxiliary results on Mal'cev algebras. In Section 3 we will then generalize a construction that Szabó used on rings in [15] to our setting to prove Theorem 1.1. 
In Section 4 we describe a new clone theoretic approach to duality that was originally suggested by Willard [17] and further developed by Davey et al. in [8]. Finally, in Section 5, we exhibit a nonabelian nilpotent expansion of $\left\langle\mathbb{Z}_{4},+\right\rangle$ with infinitely many operations that is dualizable. As far as we are aware this is the first example of a dualizable algebra that is nilpotent but nonabelian. It shows that it is really supernilpotence that prevents dualizability, not nilpotence on its own. It also demonstrates that Theorem 1.1 does not generalize to algebras of infinite type. Our example appears to be the first instance of a dualizable algebra of infinite signature, all of whose finitary nonabelian reducts with group operation are nondualizable.

Note that we will delay defining the notion of dualizability until Section 4, when technical details will become more important. We will instead provide a standard lemma giving conditions for nondualizability. We refer the reader to Clark and Davey [4] for background on natural duality.

\section{Nilpotence and supernilpotence}

We denote the set of all $k$-ary term operations on an algebra $\mathbf{A}$ by $\mathrm{Clo}_{k}(\mathbf{A})$ and call $\operatorname{Clo}(\mathbf{A}):=\bigcup_{k \in \mathbb{N}} \operatorname{Clo}_{k}(\mathbf{A})$ the clone of term operations on $\mathbf{A}[12$, Definition 4.2]. The clone of polynomial functions $\operatorname{Pol}(\mathbf{A})$ on $\mathbf{A}:=\langle A, F\rangle$ is formed by the fundamental operations $F$, the constant functions on $A$, the projections from $A^{k}$ to $A$ for $k \in \mathbb{N}$ - and all compositions thereof [12, Definition 4.4].

In [1] a stronger condition than nilpotence is defined, based on the concept of the higher commutators $\left[\alpha_{1}, \ldots, \alpha_{k}\right]$ as introduced by Bulatov [3].

Definition 2.1. [3] Let $k \geq 2$, and $\alpha_{1}, \ldots, \alpha_{k}, v$ be congruences on an algebra $\mathbf{A}$. We say that $\alpha_{1}, \ldots, \alpha_{k-1}$ centralize $\alpha_{k}$ modulo $v$ if, for all polynomial operations $f\left(\bar{x}_{1}, \ldots, \bar{x}_{k}\right)$ of $\mathbf{A}$ and tuples $\bar{a}_{1}, \ldots, \bar{a}_{k}, \bar{b}_{1}, \ldots, \bar{b}_{k}$ over $A$ that satisfy:

(1) $\bar{a}_{i} \equiv_{\alpha_{i}} \bar{b}_{i}$ for all $i \in\{1, \ldots, k\}$ and

(2) $f\left(\bar{x}_{1}, \ldots, \bar{x}_{k-1}, \bar{a}_{k}\right) \equiv_{v} f\left(\bar{x}_{1}, \ldots, \bar{x}_{k-1}, \bar{b}_{k}\right)$ for all

$$
\left(\bar{x}_{1}, \ldots, \bar{x}_{k-1}\right) \in\left(\left\{\bar{a}_{1}, \bar{b}_{1}\right\} \times \cdots \times\left\{\bar{a}_{k-1}, \bar{b}_{k-1}\right\}\right)-\left\{\left(\bar{b}_{1}, \ldots, \bar{b}_{k-1}\right)\right\},
$$

we also have

$$
f\left(\bar{b}_{1}, \ldots, \bar{b}_{k-1}, \bar{a}_{k}\right) \equiv_{v} f\left(\bar{b}_{1}, \ldots, \bar{b}_{k-1}, \bar{b}_{k}\right) .
$$

We now let the $k$-ary commutator $\left[\alpha_{1}, \ldots, \alpha_{k}\right]$ be the smallest congruence $v$ on $\mathbf{A}$ such that $\alpha_{1}, \ldots, \alpha_{k-1}$ centralize $\alpha_{k}$ modulo $v$.

One can show that for a group $\mathbf{G}$ with normal subgroups $N_{1}, \ldots, N_{k}$ the $k$-ary commutator corresponds to the product of the iterated binary commutators from classical group theory [11, Lemma 3.6]. For a ring $\mathbf{R}$ with ideals $I_{1}, \ldots, I_{k}$ the $k$ ary commutator corresponds to the ideal generated by the products of $I_{1}, \ldots, I_{k}$ in all permutations [11, Lemma 3.5].

We refer the reader to $[1,11]$ for further details on higher commutators. For our results, it is sufficient to know that the $k$-ary higher commutator is well defined and 
coincides with the term condition commutator from [9] if $k=2$ (see Lemma 2.9 below for a description of $\left[\alpha_{1}, \ldots, \alpha_{k}\right]$ specialized to nilpotent algebras).

Definition 2.2. [1] Let $k \in \mathbb{N}$, and let $\mathbf{A}$ be an algebra in a congruence permutable variety. A congruence $\alpha$ on $\mathbf{A}$ is $k$-supernilpotent if

$$
[\underbrace{\alpha, \ldots, \alpha}_{k+1}]=0,
$$

with 0 the equality relation on $A$. The algebra $\mathbf{A}$ is $k$-supernilpotent if the total relation 1 on $A$ is $k$-supernilpotent. An algebra or a congruence is supernilpotent if it is $k$-supernilpotent for some $k$.

Definition 2.3 ([9, page 124], [10, page 179]). Let $A$ be a set, and let $k \in \mathbb{N}$. Then $c: A^{k+1} \rightarrow A$ is a commutator if for all $x_{1}, \ldots, x_{k}, z \in A$,

$$
z \in\left\{x_{1}, \ldots, x_{k}\right\} \Rightarrow c\left(x_{1}, \ldots, x_{k}, z\right)=z .
$$

The commutator $c$ has rank $k$ if there exist $a_{1}, \ldots, a_{k}, o \in A: c\left(a_{1}, \ldots, a_{k}, o\right) \neq o$. Otherwise we say that it is trivial.

By [1, Lemma 7.5] an algebra $\mathbf{A}$ in a congruence permutable variety is $k$ supernilpotent if and only if $\mathbf{A}$ is nilpotent and all nontrivial commutators in Pol(A) have rank at most $k$ (see also Lemma 2.9 below).

Theorem 2.4. Let A be a finite nilpotent algebra of finite type that generates a congruence modular variety. Then the following are equivalent.

(1) A is a direct product of algebras of prime power order.

(2) There exists $M$ such that every nontrivial commutator in $\mathrm{Clo}(\mathbf{A})$ has rank at most $M$.

(3) A is supernilpotent.

Proof. (1) $\Rightarrow$ (2) follows from Freese and McKenzie [9, Theorem 14.16]. (2) $\Rightarrow$ (1) is due to Kearnes [10, Theorem 3.14]. For a proof of $(1) \Leftrightarrow(3)$ see Aichinger and Mudrinski [1, Lemma 7.6].

Lemma 2.5. Let $\mathbf{A}$ be an algebra with Mal'cev term operation $m$, let $\alpha$ be a central congruence of $\mathbf{A}$, and let $a, b, c, o \in A$ with $(c, o) \in \alpha$. Then

$$
m(m(a, o, b), o, c)=m(a, o, m(b, o, c))=m(m(a, o, c), o, b)
$$

and

$$
m(a, c, o)=m(a, o, m(o, c, o)) .
$$

Proof. As $[\alpha, 1]=0,[9$, Proposition 5.7] implies that

$$
\begin{aligned}
& m\left(m\left(a_{1}, a_{2}, a_{3}\right), m\left(b_{1}, b_{2}, b_{3}\right), m\left(c_{1}, c_{2}, c_{3}\right)\right) \\
& \quad=m\left(m\left(a_{1}, b_{1}, c_{1}\right), m\left(a_{2}, b_{2}, c_{2}\right), m\left(a_{3}, b_{3}, c_{3}\right)\right)
\end{aligned}
$$


whenever $b_{i} \equiv_{\alpha} c_{i}$ for all $i \in\{1,2,3\}$. Hence

$$
\begin{aligned}
m(m(a, o, b), o, c) & =m(m(a, o, b), m(o, o, o), m(o, o, c)) \\
& =m(m(a, o, o), m(o, o, o), m(b, o, c))=m(a, o, m(b, o, c)), \\
m(m(a, o, b), o, c) & =m(m(a, o, b), m(o, o, o), m(c, o, o)) \\
& =m(m(a, o, c), m(o, o, o), m(b, o, o))=m(m(a, o, c), o, b),
\end{aligned}
$$

and

$$
\begin{aligned}
m(a, c, o) & =m(m(a, o, o), m(c, c, c), m(c, c, o)) \\
& =m(m(a, c, c), m(o, c, c), m(o, c, o))=m(a, o, m(o, c, o)) .
\end{aligned}
$$

Lemma 2.6 [9, Lemma 7.3, Corollary 7.4]. Let A be a nilpotent algebra with Mal'cev term $m$. Then there exists $f \in \mathrm{Clo}_{3}(\mathbf{A})$ such that for all $x, b, c \in A$,

$$
m(f(x, b, c), b, c)=x \quad \text { and } \quad f(m(x, b, c), b, c)=x .
$$

In particular, for all $b, c \in A$ the map $t: A \rightarrow A$ with $x \mapsto m(x, b, c)$ is a bijection.

In the proof of Theorem 1.1 we will need a more explicit version of [9, Lemma 14.6] describing term operations on nilpotent algebras which we will state next.

Set $\underline{k}:=\{1, \ldots, k\}$, and for $x \in A^{k}$ and $S \subseteq \underline{k}$ let

$$
x_{S}:=\left(x_{i}\right)_{i \in S}
$$

Lemma 2.7 [9, see Lemma 14.6]. Let A be a finite nilpotent algebra with Mal'cev term $m$, let $k \in \mathbb{N}$, and fix a linear order $\leq$ on the power set of $\underline{k}$.

Let $f \in \mathrm{Clo}_{k+1}(\mathbf{A})$. Then for every $S \subseteq \underline{k}$ with $S \neq \emptyset$ there exists a commutator $c_{S} \in \mathrm{Clo}_{|S|+1}(\mathbf{A})$ such that for all $x \in A^{k}$ and $\bar{z} \in A$,

$$
f(x, z)=f(z, \ldots, z, z)+\sum_{S \subseteq \underline{k}, S \neq \emptyset} c_{S}\left(x_{S}, z\right)
$$

where the sum is taken with respect to $a+{ }_{z} b:=m(a, z, b)$, associated left to right, and ordered with respect to $\leq$.

Proof. In the following, all additions refer to $+_{z}$, and all sums are associated and ordered as in the statement of the lemma. Let 1 denote the total relation on $A$, and let 0 denote the equality relation on $A$. Let $(1,1]^{0}:=1$ and $(1,1]^{n+1}:=\left[1,(1,1]^{n}\right]$ for $n \in \mathbb{N}_{0}$.

First we fix $n \in \mathbb{N}$ and consider an operation $e \in \mathrm{Clo}_{k+1}(\mathbf{A})$ such that for all $x \in A^{k}$ and $z \in A$,

$$
e(x, z) \equiv z \quad \bmod (1,1]^{n} \quad \text { and } \quad e(z, \ldots, z, z)=z
$$


We claim that there exist commutators $d_{S} \in \mathrm{Clo}_{|S|+1}(\mathbf{A}), S \subseteq \underline{k}, S \neq \emptyset$, such that for all $x \in A^{k}$ and $z \in A$,

$$
e(x, z) \equiv \sum_{S \subseteq \underline{k}, S \neq \emptyset} d_{S}\left(x_{S}, z\right) \quad \bmod (1,1]^{n+1} \quad \text { and } \quad d_{S}\left(x_{S}, z\right) \equiv z \quad \bmod (1,1]^{n} .
$$

We prove this by induction on $k$. If $k=1$, then $e$ itself is a commutator, and the assertion is trivially true. Assume $k>1$ in the following. Let $e_{o}:=e$, and for $j \in \underline{k}$ define $e_{j} \in \mathrm{Clo}_{k+1}(\mathbf{A})$ iteratively by

$$
e_{j}\left(x_{1}, \ldots, x_{k}, z\right):=m\left(e_{j-1}\left(x_{1}, \ldots, x_{k}, z\right), e_{j-1}\left(x_{1}, \ldots, x_{j-1}, z, x_{j+1}, \ldots, x_{k}, z\right), z\right) .
$$

Then $e_{j}(x, z) \equiv z \bmod (1,1]^{n}$ for all $x \in A^{k}, z \in A$, and $e_{j}\left(x_{1}, \ldots, x_{k}, z\right)=z$ whenever $x_{l}=z$ for some $l \leq j$. In particular, $e_{k}$ is a commutator.

For $T \subseteq \underline{k}$ define $\delta_{T}: A^{k+1} \rightarrow A^{k+1}$ by

$$
\left(\delta_{T}\left(x_{1}, \ldots, x_{k}, z\right)\right)_{i}:= \begin{cases}x_{i} & \text { if } i \in T \\ z & \text { otherwise }\end{cases}
$$

For $a, z \in A$ write ${ }_{z} a:=m(z, a, z)$. Note that $(1,1]^{n} /(1,1]^{n+1}$ is central in $\mathbf{A} /(1,1]^{n+1}$. Hence, for every $z \in A$, the operations $+_{z},-{ }_{z}$, and $z$ induce addition, inverse, and zero element of an abelian group on $\left\{a /(1,1]^{n+1}: a \equiv z \bmod (1,1]^{n}\right\}$ by Lemma 2.5 . From the definitions and Lemma 2.5 it is straightforward that for all $x \in A^{k}$ and $z \in A$,

$$
e_{k}(x, z) \equiv \sum_{T \subseteq \underline{k}}(-1)^{k-|T|} e\left(\delta_{T}(x, z)\right) \bmod (1,1]^{n+1}
$$

with - referring to $-_{z}$ and the order of the sum irrelevant because the summands commute $\left(\bmod (1,1]^{n+1}\right)$ with respect to $+_{z}$. Since $e \circ \delta_{\underline{k}}=e$, we obtain for all $x \in A^{k}$ and $z \in A$,

$$
e(x, z) \equiv e_{k}(x, z)+\sum_{T \subset \underline{k}}(-1)^{k+1-|T|} e\left(\delta_{T}(x, z)\right) \bmod (1,1]^{n+1} .
$$

Let $T \subset \underline{k}$. Then $e \circ \delta_{T}$ does not depend on $x_{i}$ for $i \in \underline{k} \backslash T$. From the induction assumption for $k-1$ it follows that we have commutators $d_{S}^{T} \in \mathrm{Clo}_{|S|+1}(\mathbf{A})$, for $S \subseteq$ $T, S \neq \emptyset$, such that for all $x \in A^{k}$ and $z \in A$,

$$
e\left(\delta_{T}(x, z)\right) \equiv \sum_{S \subseteq T, S \neq \emptyset} d_{S}^{T}\left(x_{S}, z\right) \quad \bmod (1,1]^{n+1}, \quad d_{S}^{T}\left(x_{S}, z\right) \equiv z \quad \bmod (1,1]^{n} .
$$

Hence for every $x \in A^{k}$ and $z \in A$,

$$
\begin{aligned}
\sum_{T \subset \underline{k}}(-1)^{k+1-|T|} e\left(\delta_{T}(x, z)\right) & \equiv \sum_{T \subset \underline{k}}(-1)^{k+1-|T|} \sum_{S \subseteq T, S \neq \emptyset} d_{S}^{T}\left(x_{S}, z\right) \bmod (1,1]^{n+1} \\
& \equiv \sum_{S \subset \underline{k}, S \neq \emptyset} \underbrace{\sum_{S \subseteq T \subset \underline{k}}(-1)^{k+1-|T|} d_{S}^{T}\left(x_{S}, z\right)}_{=: d_{S}\left(x_{S}, z\right)} \bmod (1,1]^{n+1} .
\end{aligned}
$$


We observe that $d_{S} \in \mathrm{Clo}_{|S|}(\mathbf{A})$ is a commutator because $d_{S}^{T}$ is a commutator for every $S \subseteq T \subset \underline{k}$ and that $d_{S}\left(x_{S}, z\right) \equiv z \bmod (1,1]^{n}$ for every $x \in A^{k}, z \in A$. Finally, (2.2) yields

$$
e(x, z) \equiv \underbrace{e_{k}(x, z)}_{=: d_{\underline{k}}(x, z)}+\sum_{S \subset \underline{k}, S \neq \emptyset} d_{S}\left(x_{S}, z\right) \bmod (1,1]^{n+1}
$$

which proves (2.1).

Next we consider an arbitrary operation $f \in \mathrm{Clo}_{k+1}$ (A). Let $n \in \mathbb{N}_{0}$. We claim that we have commutators $c_{S} \in \mathrm{Clo}_{|S|+1}(\mathbf{A})$ for $S \subseteq \underline{k}, S \neq \emptyset$, such that for all $x \in A^{k}$ and $z \in A$,

$$
f(x, z) \equiv f(z, \ldots, z, z)+\sum_{S \subseteq \underline{k}, S \neq \emptyset} c_{S}\left(x_{S}, z\right) \bmod (1,1]^{n} .
$$

For the proof we use induction on $n$. For $n=0$ the statement is trivially true. So assume that (2.3) holds for some fixed $n \geq 0$. By Lemma 2.6 there exists $e \in \mathrm{Clo}_{k+1}(\mathbf{A})$ such that for all $x \in A^{k}$ and $z \in A$,

$$
f(x, z)=f(z, \ldots, z, z)+\sum_{S \subseteq \underline{k}, S \neq \emptyset} c_{S}\left(x_{S}, z\right)+e(x, z)
$$

as well as $e(x, z) \equiv z \bmod (1,1]^{n}$ and $e(z, \ldots, z, z)=z$. Now $e$ can be written as a sum of commutators $d_{S}$ modulo $(1,1]^{n+1}$ as in (2.1). Then (2.1) and (2.4) yield

$$
f(x, z) \equiv f(z, \ldots, z, z)+\sum_{S \subseteq \underline{k}, S \neq \emptyset} c_{S}\left(x_{S}, z\right)+\sum_{S \subseteq \underline{k}, S \neq \emptyset} d_{S}\left(x_{S}, z\right) \bmod (1,1]^{n+1} .
$$

From Lemma 2.5 we obtain

$$
f(x, z) \equiv f(z, \ldots, z, z)+\sum_{S \subseteq \underline{k}, S \neq \emptyset}\left(c_{S}\left(x_{S}, z\right)+d_{S}\left(x_{S}, z\right)\right) \bmod (1,1]^{n+1} .
$$

Since $c_{S}+d_{S}$ is a commutator for every $S$, the induction step is proved. Thus we can represent $f$ as in (2.3) for every $n \in \mathbb{N}_{0}$. For $N$ such that $(1,1]^{N}=0$ this yields the lemma.

We conclude this section with three observations on commutator polynomials and higher commutators of congruences.

Lemma 2.8. Let $\mathbf{A}$ be an algebra in a congruence modular variety, let $k \geq 2$, and let $c \in \mathrm{Clo}_{k+1}(\mathbf{A})$ be a commutator. Let $\alpha, \beta$ be congruences of $\mathbf{A}$, and let $a_{1}, \ldots, a_{k}, o \in A$ such that $a_{1} \equiv_{\alpha} o, a_{2} \equiv_{\beta}$ o. Then

$$
c\left(a_{1}, \ldots, a_{k}, o\right) \equiv_{[\alpha, \beta]} o .
$$

Proof. Consider $M_{\mathbf{A}}(\alpha, \beta)$, the subuniverse of $\mathbf{A}^{2 \times 2}$ that is generated by all the elements

$$
\left(\begin{array}{ll}
a & b \\
a & b
\end{array}\right),\left(\begin{array}{ll}
e & e \\
f & f
\end{array}\right) \quad \text { for } a, b, e, f \in A \text { with } a \equiv_{\alpha} b, e \equiv_{\beta} f .
$$


Then

$$
c\left(\left(\begin{array}{cc}
o & a_{1} \\
o & a_{1}
\end{array}\right),\left(\begin{array}{cc}
o & o \\
a_{2} & a_{2}
\end{array}\right),\left(\begin{array}{ll}
a_{3} & a_{3} \\
a_{3} & a_{3}
\end{array}\right), \ldots,\left(\begin{array}{ll}
a_{k} & a_{k} \\
a_{k} & a_{k}
\end{array}\right),\left(\begin{array}{cc}
o & o \\
o & o
\end{array}\right)\right)=\left(\begin{array}{cc}
o & o \\
o & c\left(a_{1}, \ldots, a_{k}, o\right)
\end{array}\right)
$$

is contained in $M_{\mathbf{A}}(\alpha, \beta)$ and the result follows from the definition of the term condition commutator.

LEMMA 2.9. Let $\mathbf{A}$ be a nilpotent algebra generating a congruence modular variety, let $k \in \mathbb{N}$, and let $\alpha_{1}, \ldots, \alpha_{k}$ be congruences of $\mathbf{A}$. Then $\left[\alpha_{1}, \ldots, \alpha_{k}\right]$ is the congruence of $\mathbf{A}$ that is generated by

$$
\begin{gathered}
T:=\left\{\left(c\left(a_{1}, \ldots, a_{k}, o\right), o\right): c \in \operatorname{Pol}_{k+1}(\mathbf{A}), c\right. \text { is a commutator } \\
\left.\left(a_{1}, o\right) \in \alpha_{1}, \ldots,\left(a_{k}, o\right) \in \alpha_{k}\right\} .
\end{gathered}
$$

Proof. Let $\left(o_{1}, \ldots, o_{k}\right) \in A^{k}$. As in [1, Definition 4.9] we say that $f: A^{k} \rightarrow A$ is absorbing at $\left(o_{1}, \ldots, o_{k}\right)$ if for all $\left(x_{1}, \ldots, x_{k}\right) \in A^{k}$,

$$
\left(\exists i \in \underline{k}: x_{i}=o_{i}\right) \Rightarrow f\left(x_{1}, \ldots, x_{k}\right)=f\left(o_{1}, \ldots, o_{k}\right) .
$$

By $\left[1\right.$, Lemma 6.9] $\left[\alpha_{1}, \ldots, \alpha_{k}\right]$ is the congruence of $\mathbf{A}$ that is generated by

$$
\begin{gathered}
R:=\left\{\left(f\left(b_{1}, \ldots, b_{k}\right), f\left(o_{1}, \ldots, o_{k}\right)\right): f \in \operatorname{Pol}_{k}(\mathbf{A}), f\right. \text { is absorbing at } \\
\left.\left(o_{1}, \ldots, o_{k}\right), \text { and }\left(b_{1}, o_{1}\right) \in \alpha_{1}, \ldots,\left(b_{k}, o_{k}\right) \in \alpha_{k}\right\} .
\end{gathered}
$$

Hence it suffices to prove that

$$
T=R \text {. }
$$

For the inclusion $\subseteq$ let $(a, o) \in T$. Then we have a commutator $c$ in $\operatorname{Pol}_{k+1}(\mathbf{A})$, and $\left(a_{1}, o\right) \in \alpha_{1}, \ldots,\left(a_{k}, o\right) \in \alpha_{k}$ such that $\left(c\left(a_{1}, \ldots, a_{k}, o\right), o\right)=(a, o)$.

Consider $f: A^{k} \rightarrow A,\left(x_{1}, \ldots, x_{k}\right) \mapsto c\left(x_{1}, \ldots, x_{k}, o\right)$. Then $f$ is a $k$-ary polynomial function on $\mathbf{A}$ that is absorbing at $(o, \ldots, o)$. Since

$$
\left(f\left(a_{1}, \ldots, a_{k}\right), f(o, \ldots, o)\right)=(a, o),
$$

we obtain $(a, o) \in R$.

For the converse inclusion $\supseteq$ in $(2.5)$, let $(a, o) \in R$. Then we have $\left(b_{1}, o_{1}\right) \in$ $\alpha_{1}, \ldots,\left(b_{k}, o_{k}\right) \in \alpha_{k}$ and some $f \in \operatorname{Pol}_{k}(\mathbf{A})$ that is absorbing at $\left(o_{1}, \ldots, o_{k}\right)$ such that $\left(f\left(b_{1}, \ldots, b_{k}\right), f\left(o_{1}, \ldots, o_{k}\right)\right)=(a, o)$. Since $\mathbf{A}$ is nilpotent, it has a Mal'cev term operation $m$ by [9, Theorem 6.2]. Define a $(k+1)$-ary polynomial operation $c$ on A by

$$
c\left(x_{1}, \ldots, x_{k}, z\right):=m\left(f\left(m\left(x_{1}, z, o_{1}\right), \ldots, m\left(x_{k}, z, o_{k}\right)\right), o, z\right) .
$$

Note that $c$ is a commutator. Let $i \in \underline{k}$. By Lemma 2.6, we have $a_{i} \in A$ such that $m\left(a_{i}, o, o_{i}\right)=b_{i}$. Note that $o_{i} \equiv_{\alpha_{i}} b_{i}$ implies $a_{i} \equiv_{\alpha_{i}} o$. Now $c\left(a_{1}, \ldots, a_{k}, o\right)=$ $f\left(b_{1}, \ldots, b_{k}\right)$ and $(a, o) \in T$. 
Let $m$ be a Mal'cev term operation on an algebra A. Under certain conditions commutator terms on $\mathbf{A}$ turn out to be multilinear and alternating with respect to the operation $a+{ }_{z} b:=m(a, z, b)$.

Lemma 2.10. Let A be a nilpotent algebra with Mal'cev term operation $m$, let $k \in \mathbb{N}$, let $c \in \mathrm{Clo}_{k+1}(\mathbf{A})$ be a commutator, and let $\alpha \in \mathrm{Con}(\mathbf{A})$ be $k$-supernilpotent.

(1) Then for all $x_{1}, \ldots, x_{k}, y, z \in A$ with $\left(x_{1}, z\right), \ldots,\left(x_{k}, z\right),(y, z) \in \alpha$,

$$
c\left(x_{1}, \ldots, x_{i}+_{z} y, \ldots, x_{k}, z\right)=c\left(x_{1}, \ldots, x_{i}, \ldots, x_{k}, z\right)+_{z} c\left(x_{1}, \ldots, y, \ldots, x_{k}, z\right)
$$

(multilinearity).

(2) Let $i, j \in\{1, \ldots, k\}$ be distinct. Assume that for all $x_{1}, \ldots, x_{k}, z \in A$ with $\left(x_{1}, z\right), \ldots,\left(x_{k}, z\right) \in \alpha, x_{i}=x_{j} \Rightarrow c\left(x_{1}, \ldots, x_{k}, z\right)=z$. Then for all $x_{1}, \ldots, x_{k}, z \in$ $A$ with $\left(x_{1}, z\right), \ldots,\left(x_{k}, z\right) \in \alpha$,

$$
\begin{array}{r}
c\left(x_{1}, \ldots, x_{i}, \ldots, x_{j}, \ldots, x_{k}, z\right)+{ }_{z} c\left(x_{1}, \ldots, \underset{j}{x_{j}}, \ldots, \underset{j}{x_{i}}, \ldots, x_{k}, z\right)=z \\
\text { (alternating) } .
\end{array}
$$

Proof. For simplicity, we will write + instead of $+_{z}$ throughout the proof. For (1) define

$$
\begin{aligned}
d\left(x_{1}, \ldots, x_{k}, y, z\right):= & m\left(c\left(x_{1}, \ldots, x_{i}+y, \ldots, x_{k}, z\right),\right. \\
& \left.\quad c\left(x_{1}, \ldots, x_{i}, \ldots, x_{k}, z\right)+c\left(x_{1}, \ldots, \underset{i}{y}, \ldots, x_{k}, z\right), z\right) .
\end{aligned}
$$

Then $d$ is a commutator. Let $x_{1}, \ldots, x_{k}, y, z \in A$ be fixed such that $\left(x_{1}, z\right), \ldots$, $\left(x_{k}, z\right),(y, z) \in \alpha$. Since $[\underbrace{\alpha, \ldots, \alpha}_{k+1}]=0, d\left(x_{1}, \ldots, x_{k}, y, z\right)=z$ by Lemma 2.9. Since

$$
m\left(c\left(\ldots, x_{i}, \ldots\right)+c\left(\ldots, y_{i}, \ldots\right), c\left(\ldots, x_{i}, \ldots\right)+c\left(\ldots, y_{i}, \ldots\right), z\right)=z,
$$

Lemma 2.6 yields $c\left(\ldots, x_{i}+y, \ldots\right)=c\left(\ldots, x_{i}, \ldots\right)+c(\ldots, y, \ldots)$.

For (2) we note that (1) implies

$$
\begin{aligned}
& c\left(\ldots, x_{i}+x_{j}, \ldots, x_{i}+x_{j}, \ldots\right)=c\left(\ldots, x_{i}, \ldots, x_{i}, \ldots\right)+c\left(\ldots, x_{i}, \ldots, x_{j}, \ldots\right) \\
& \quad+c\left(\ldots, x_{j}, \ldots, x_{i}, \ldots\right)+c\left(\ldots, x_{j}, \ldots, x_{j}, \ldots\right) .
\end{aligned}
$$

\section{Proof of Theorem 1.1}

This section consists almost entirely of the proof of the following result which will eventually yield Theorem 1.1.

Theorem 3.1. Let $\mathbf{A}$ be a finite nilpotent algebra in a congruence modular variety. Assume that A has a nonabelian k-supernilpotent congruence $\alpha$ (such that $[\underbrace{\alpha, \ldots, \alpha}_{k+1}]=0)$ for some $k \geq 2$. Then $\mathbf{A}$ is inherently nondualizable, that is, every finite superalgebra $\mathbf{B}$ of $\mathbf{A}$ is nondualizable. 
Let $\mathbf{A}$ and $\mathbf{B}$ satisfy the assumptions of Theorem 3.1. We will show that $\mathbf{B}$ is not dualizable using the ghost element method in the form of the following lemma.

Lemma 3.2 (Nondualizability [5, Lemma 5.2]). Let $\mathbf{B}$ be a finite algebra and let $N \in$ $\mathbb{N}$. Assume that there is a subalgebra $\mathbf{D}$ of $\mathbf{B}^{J}$, for some set $J$, and an infinite subset $D_{0}$ of $D$ such that:

(1) for each homomorphism $\varphi: \mathbf{D} \rightarrow \mathbf{B}$, the equivalence relation $\operatorname{ker}\left(\left.\varphi\right|_{D_{0}}\right)$ has a unique block of size greater than $N$; and

(2) the algebra $\mathbf{D}$ does not contain the element $g$ of $B^{J}$ given by $g(i):=b_{i}(i)$, where $i \in J$ and $b_{i}$ is any element of the unique infinite block of $\operatorname{ker}\left(\left.\pi_{i}\right|_{D_{0}}\right)$.

Then $\mathbf{B}$ is nondualizable.

Since $\mathbf{A}$ is nilpotent, it has a Mal'cev term operation $m$ by [9, Theorem 6.2]. By assumption there exist a nonabelian congruence $\beta$ of $\mathbf{A}$ and some $k^{\prime} \geq 2$ such that $[\underbrace{\beta, \ldots, \beta}_{k^{\prime}+1}]=0$. Let $\alpha \in \operatorname{Con}(\mathbf{A})$ be minimal such that $\alpha \leq \beta$ and $\alpha$ is nonabelian.

Since $\mathbf{A}$ is nilpotent, $\gamma:=[\alpha, \alpha]$ is strictly smaller than $\alpha$ and consequently abelian. Further, we have $2 \leq k \leq k^{\prime}$ such that $[\underbrace{\alpha, \ldots, \alpha}_{k+1}]=0$ but $[\underbrace{\alpha, \ldots, \alpha}_{k}] \neq 0$. Hence, by Lemma 2.9, there exist a commutator $c \in \operatorname{Pol}_{k+1}(\mathbf{A})$ and $\left(x_{1}, z\right), \ldots,\left(x_{k}, z\right) \in \alpha$ such that $c\left(x_{1}, \ldots, x_{k}, z\right) \neq z$. We will distinguish the following two cases.

Case 1. All commutators $c \in \operatorname{Pol}(\mathbf{A})$ of rank $k$ satisfy $c\left(x_{1}, \ldots, x_{k}, z\right)=z$ whenever $x_{i}=x_{j}$ for some $i \neq j$ and $\left(x_{1}, z\right), \ldots,\left(x_{k}, z\right) \in \alpha$. Fix a commutator $f \in \operatorname{Pol}_{k+1}(\mathbf{A})$, and fix $o \in A,\left(a_{1}, o\right), \ldots,\left(a_{k}, o\right) \in \alpha$ such that $f\left(a_{1}, \ldots, a_{k}, o\right) \neq o$.

Case 2. There exists a commutator $c \in \operatorname{Pol}(\mathbf{A})$ of rank $k$ and $\left(x_{1}, z\right), \ldots,\left(x_{k}, z\right) \in \alpha$ such that $x_{i}=x_{j}$ for some $i \neq j$ and $c\left(x_{1}, \ldots, x_{k}, z\right) \neq z$. By permuting coordinates if necessary, we then also have a commutator $f \in \operatorname{Pol}_{k+1}(\mathbf{A})$ of rank $k$ and $o \in$ $A,\left(a_{1}, o\right), \ldots,\left(a_{k}, o\right) \in \alpha$ with $a_{1}=a_{2}$ such that $f\left(a_{1}, \ldots, a_{k}, o\right) \neq o$.

With elements $a_{1}, \ldots, a_{k}, o$ and commutator $f$ chosen according to the above cases we proceed to construct the subuniverse $D$ of $\left(\mathbf{B}^{\mathcal{P}(\underline{k})}\right)^{\mathbb{Z}}$ (where $\mathcal{P}(\underline{k})$ is the power set of $\underline{k}$ ).

For $a \in A$, let $\bar{a} \in A^{\mathcal{P}(\underline{k})}$ such that $\bar{a}(S):=a$ for all $S \subseteq \underline{k}$. For $i \in \underline{k}$, define $u_{i} \in A^{\mathcal{P}(\underline{k})}$ by

$$
u_{i}(S):= \begin{cases}a_{i} & \text { if } i \in S, \\ o & \text { otherwise. }\end{cases}
$$

Let $t:=2|B|+1$. For $i \in \mathbb{Z}$ let $d_{i} \in\left(A^{\mathcal{P}(\underline{k})}\right)^{\mathbb{Z}}$ be given by

$$
d_{i}(j):= \begin{cases}u_{1} & \text { if } j \in\{i, i+t+3\}, \\ u_{2} & \text { if } j \in\{i+1, i+t+2\}, \\ \bar{o} & \text { else. }\end{cases}
$$

Then

$$
d_{i}=(\ldots, \bar{o}, u_{1}, u_{2}, \underbrace{\bar{o}, \ldots, \bar{o}}_{t}, u_{2}, u_{1}, \bar{o}, \ldots) .
$$


For $l \in\{3, \ldots, k\}$ let

$$
c_{l}:=\left(\ldots, u_{l}, u_{l}, \ldots\right) \in\left(A^{\mathcal{P}(\underline{k})}\right)^{\mathbb{Z}},
$$

and for $a \in A$ let

$$
\overline{\bar{a}}:=(\ldots, \bar{a}, \bar{a}, \ldots) \in\left(A^{\mathcal{P}(\underline{k})}\right)^{\mathbb{Z}},
$$

that is, $c_{l}(j)=u_{l}$ and $\overline{\bar{a}}(j)=\bar{a}$ for all $j \in \mathbb{Z}$.

We let $D$ be the subuniverse of $\left(\mathbf{B}^{\mathcal{P}(\underline{k})}\right)^{\mathbb{Z}}$ that is generated by $\left\{d_{i}: i \in \mathbb{Z}\right\} \cup$ $\left\{c_{3}, \ldots, c_{k}\right\} \cup\{\overline{\bar{a}}: a \in A\}$. Note that $D \subseteq\left(A^{\mathcal{P}(\underline{k})}\right)^{\mathbb{Z}}$.

To describe the set $D_{0}$ from Lemma 3.2 we construct yet more elements in $D$. For $i \in \mathbb{Z}$ consider

$$
\begin{aligned}
& d_{i}=\left(\ldots \bar{o} \stackrel{i-t-2}{\bar{o}} \bar{o} \bar{o} \ldots \bar{o} \stackrel{i}{u_{1}} u_{2} \bar{o} \ldots \bar{o} \stackrel{i+t+2}{u_{2}} u_{1} \bar{o} \ldots\right), \\
& d_{i-t-2}=\left(\ldots \bar{o} \quad u_{1} \quad u_{2} \bar{o} \ldots \bar{o} u_{2} u_{1} \bar{o} \ldots \bar{o} \quad \bar{o} \quad \bar{o} \quad \bar{o} \ldots\right),
\end{aligned}
$$

and define $v_{i, i+1}:=f\left(d_{i}, d_{i-t-2}, c_{3}, \ldots, c_{k}, \overline{\bar{o}}\right)$. Then

$$
v_{i, i+1}(j)= \begin{cases}f\left(u_{1}, u_{2}, u_{3}, \ldots, u_{k}, \bar{o}\right) & \text { if } j=i \\ f\left(u_{2}, u_{1}, u_{3}, \ldots, u_{k}, \bar{o}\right) & \text { if } j=i+1 \\ \bar{o} & \text { otherwise. }\end{cases}
$$

Now let $e:=f\left(u_{1}, \ldots, u_{k}, \bar{o}\right)$. Since $f$ is a commutator,

$$
e(S)= \begin{cases}f\left(a_{1}, \ldots, a_{k}, o\right) & \text { if } S=\underline{k} \\ o & \text { otherwise. }\end{cases}
$$

We recall that $\gamma=[\alpha, \alpha]$ is abelian. For $x, y \in o / \gamma$ define

$$
x+{ }_{o} y:=m(x, o, y) \text {. }
$$

Then $\left\langle o / \gamma,{ }_{o}\right\rangle$ is an abelian group by Lemma 2.5 .

Since $k \geq 2$, we have that $f\left(a_{1}, \ldots, a_{k}, o\right) \equiv_{\gamma} o$ by Lemma 2.8. Hence all entries of $f\left(u_{1}, u_{2}, u_{3}, \ldots, u_{k}, \bar{o}\right)$ are contained in $o / \gamma$. Similarly, we get that $f\left(u_{2}, u_{1}, u_{3}, \ldots, u_{k}, \bar{o}\right) \in(o / \gamma)^{\mathcal{P}(\underline{k})}$.

For Case 1, Lemma 2.10 yields

$$
f\left(a_{2}, a_{1}, a_{3}, \ldots, a_{k}, o\right)=-f\left(a_{1}, a_{2}, a_{3}, \ldots, a_{k}, o\right)
$$

in the group $\left\langle o / \gamma,+_{o}\right\rangle$. Hence

$$
v_{i, i+1}=(\ldots, \bar{o}, \underset{i}{e},-\underset{i+1}{e}, \bar{o}, \ldots) .
$$

For $i<j$ define $v_{i, j}:=\sum_{l=i}^{j-1} v_{l, l+1}$. Then

$$
v_{i, j}=(\ldots, \bar{o}, \underset{i}{e}, \bar{o}, \ldots, \bar{o}, \underset{j}{-e}, \bar{o}, \ldots) .
$$


For Case 2, from $a_{1}=a_{2}$ we obtain

$$
v_{i, i+1}=(\ldots, \bar{o}, \underset{i}{e}, \underset{i+1}{e}, \bar{o}, \ldots) .
$$

For $i<j$ let $v_{i, j}:=\sum_{l=i}^{j-1}(-1)^{l-i} v_{l, l+1}$, which yields

$$
v_{i, j}=\left(\ldots, \bar{o}, \underset{i}{e}, \bar{o}, \ldots, \bar{o},(-1)_{j}^{j-i-1} e, \bar{o}, \ldots\right) .
$$

We want to use Lemma 3.2 with $D_{0}:=\left\{v_{0, i}: i \in \mathbb{N}\right\}, N:=2|B|(2|B|-1)$, and the ghost element $g$ with $g(0):=e$ and $g(i):=\bar{o}$ for $i \neq 0$. We will first establish the second condition of the lemma, that is, that the ghost is not in $D$. This still requires some more preparation.

First we observe that if an element in $D$ is congruent to $\overline{\bar{o}}$ modulo $\gamma$, then it can be written as sum of a constant and commutators evaluated at generators of $\mathbf{D}$ such that every summand is congruent to $\overline{\bar{o}}$ modulo $\gamma$.

Lemma 3.3. Let $l<r, n:=r-l+k-1$, and let $h \in \operatorname{Pol}_{n+1}(\mathbf{A})$ such that

$$
h\left(d_{l}, d_{l+1}, \ldots, d_{r}, c_{3}, \ldots, c_{k}, \overline{\bar{o}}\right) \equiv_{\gamma} \overline{\bar{o}}
$$

For $S \subseteq \underline{n}, S \neq \emptyset$, let $c_{S} \in \operatorname{Pol}_{|S|+1}(\mathbf{A})$ be commutators such that for all $x \in A^{n}$ and $z \in A$,

$$
h(x, z)=h(z, \ldots, z, z)+\sum_{S \subseteq \underline{n}, S \neq \emptyset} c_{S}\left(x_{S}, z\right) .
$$

Write $g_{1}:=d_{l}, g_{2}:=d_{l+1}, \ldots, g_{r-l+1}:=d_{r}, g_{r-l+2}:=c_{3}, \ldots, g_{n}:=c_{k}$. Then $h(\overline{\bar{o}}, \ldots, \overline{\bar{o}})$ $\equiv_{\gamma} \overline{\bar{o}}$ and for all $S \subseteq \underline{n}$ with $S \neq \emptyset$,

$$
c_{S}\left(g_{S}, \overline{\bar{o}}\right) \equiv_{\gamma} \overline{\bar{o}}
$$

PROOF. We will once again write + instead of $+_{z}$. We have

$$
\begin{aligned}
g_{1} & =\left(\begin{array}{llllllllllll}
\ldots & u_{1} & u_{2} & \bar{o} & \ldots & u_{2} & u_{1}+t+3 & \bar{o} & \ldots & \bar{o} & \bar{o} & \ldots
\end{array}\right), \\
g_{2} & =\left(\begin{array}{llllllllllll}
\ldots & \bar{o} & u_{1} & u_{2} & \ldots & \bar{o} & u_{2} & u_{1} & \ldots & \bar{o} & \bar{o} & \ldots
\end{array}\right), \\
& \vdots \\
g_{t+4} & =\left(\begin{array}{lllllllllllll}
\ldots & \bar{o} & \bar{o} & \bar{o} & \ldots & \bar{o} & u_{1} & u_{2} & \ldots & u_{2} & u_{1} & \ldots
\end{array}\right),
\end{aligned}
$$

Note that $\left(h\left(g_{1}, \ldots, g_{n}, \overline{\bar{o}}\right)\right)(l)(\emptyset)=h(o, \ldots, o)$ implies $h(\overline{\bar{o}}, \ldots, \overline{\bar{o}}) \equiv_{\gamma} \overline{\bar{o}}$. If $|S| \geq 2$, then $c_{S}\left(g_{S}, \overline{\bar{o}}\right) \equiv_{\gamma} \overline{\bar{o}}$ by Lemma 2.8. So it only remains to prove the assertion for $c_{S}$ with $|S|=1$. First we claim that

$$
c_{\{s\}}\left(a_{1}, o\right) \equiv_{\gamma} o \quad \text { for all } s \in\{1, \ldots, r-l+1\} .
$$


For the proof we use induction on $s$. For $s \in\{1, \ldots, t+3\}$,

$$
\left(h\left(g_{1}, \ldots, g_{n}, \overline{\bar{o}}\right)\right)(l-1+s)(\{1\})=h(o, \ldots, o)+c_{\{s\}}\left(a_{1}, o\right)
$$

and consequently $c_{\{s\}}\left(a_{1}, o\right) \equiv_{\gamma} o$. Now let $s \in\{t+4, \ldots, r-l+1\}$. Then

$$
\left(h\left(g_{1}, \ldots, g_{n}, \overline{\bar{o}}\right)\right)(l-1+s)(\{1\})=h(o, \ldots, o)+c_{\{s-(t+3)\}}\left(a_{1}, o\right)+c_{\{s\}}\left(a_{1}, o\right) .
$$

Since $c_{\{s-(t+3)\}}\left(a_{1}, o\right) \equiv_{\gamma} o$ by the induction assumption, we get $c_{\{s\}}\left(a_{1}, o\right) \equiv_{\gamma} o$ as well. Hence (3.1) is proved.

Similarly we obtain $c_{\{s\}}\left(a_{2}, o\right) \equiv_{\gamma} o$, which together with (3.1) implies $c_{\{s\}}\left(g_{s}, \overline{\bar{o}}\right) \equiv_{\gamma}$ $\overline{\bar{o}}$ for all $s \in\{1, \ldots, r-l+1\}$.

Now let $j \in\{3, \ldots, k\}$. Then

$$
\left(h\left(g_{1}, \ldots, g_{n}, \overline{\bar{o}}\right)\right)(l)(\{j\})=h(o, \ldots, o)+c_{\{r-l+j-1\}}\left(a_{j}, o\right),
$$

which yields $c_{\{s\}}\left(g_{s}, \overline{\bar{o}}\right) \equiv_{\gamma} \overline{\bar{o}}$ for all $s \in\{r-l+2, \ldots, n\}$.

We are now ready to characterize those elements in $D$ that are congruent to $\overline{\bar{o}}$ modulo $\gamma$ by their parities.

Lemma 3.4. Let $w \in D$ be such that $w(i)(S) \equiv_{\gamma}$ o for all $i \in \mathbb{Z}, S \in \mathcal{P}(\underline{k})$. Assume that $w(i)=\bar{o}$ for all but finitely many $i \in \mathbb{Z}$. Then the following parity conditions hold in the abelian group $\left\langle o / \gamma,+_{o}\right\rangle$ : in Case 1 ,

$$
\sum_{i \in \mathbb{Z}} \sum_{S \subseteq \underline{k}}(-1)^{|S|} w(i)(S)=o ;
$$

in Case 2,

$$
\sum_{i \in \mathbb{Z}}(-1)^{i} \sum_{S \subseteq \underline{k}}(-1)^{|S|} w(i)(S)=o .
$$

Proof. By Lemmas 2.7 and 3.3, $w$ is a sum of elements $c\left(g_{1}, \ldots, g_{n}, \overline{\bar{o}}\right)$ where $c \in \operatorname{Pol}_{n}(\mathbf{A})$ is a commutator and $\left\{g_{1}, \ldots, g_{n}\right\} \subseteq\left\{d_{i}: i \in \mathbb{Z}\right\} \cup\left\{c_{3}, \ldots, c_{k}\right\}$. Since $[\underbrace{\alpha, \ldots, \alpha}_{k+1}]=0$, we may assume that $n \leq k$ (for otherwise $c\left(g_{1}, \ldots, g_{n}, \overline{\bar{o}}\right)=\overline{\bar{o}}$ by

Lemma 2.9). Further, by Lemma 3.3, all these summands are congruent to $\overline{\bar{o}}$ modulo $\gamma$. Since $t_{o}$ is commutative on $o / \gamma$, it suffices to prove the assertion for every summand. So assume that $w=c\left(g_{1}, \ldots, g_{n}, \overline{\bar{o}}\right)$ where $c \in \operatorname{Pol}_{n}(\mathbf{A})$ is a commutator and $\left\{g_{1}, \ldots, g_{n}\right\} \subseteq\left\{d_{i}: i \in \mathbb{Z}\right\} \cup\left\{c_{3}, \ldots, c_{k}\right\}$.

Suppose that $\left\{g_{1}(i), \ldots, g_{n}(i), \bar{o}\right\} \neq\left\{u_{1}, \ldots, u_{k}, \bar{o}\right\}$ for all $i \in \mathbb{Z}$. We claim that

$$
\forall i \in \mathbb{Z}, \quad \sum_{S \subseteq \underline{k}}(-1)^{|S|} w(i)(S)=o .
$$

For $i \in \mathbb{Z}$ fixed, let $l \in \underline{k}$ be such that $u_{l} \notin\left\{g_{1}(i), \ldots, g_{n}(i)\right\}$. Let $S \subseteq \underline{k} \backslash\{l\}$. Since $u_{j}(S)=u_{j}(S \cup\{l\})$ for all $j \in \underline{k}$ with $j \neq l$,

$$
w(i)(S)=\left(c\left(g_{1}, \ldots, g_{n}, o\right)\right)(i)(S)=\left(c\left(g_{1}, \ldots, g_{n}, o\right)\right)(i)(S \cup\{l\})=w(i)(S \cup\{l\})
$$

From this, (3.2) and the result follow. 
Now suppose that $i \in \mathbb{Z}$ such that $\left\{g_{1}(i), \ldots, g_{n}(i)\right\}=\left\{u_{1}, \ldots, u_{k}\right\}$. Then $n=k$. Without loss of generality we may assume that $g_{j}(i)=u_{j}$ for all $j \in \underline{k}$. Then

$$
\begin{aligned}
g_{1} & \in\left\{d_{i}, d_{i-t-3}\right\}, \\
g_{2} & \in\left\{d_{i-1}, d_{i-t-2}\right\}, \\
g_{3} & =c_{3}, \\
& \vdots \\
g_{k} & =c_{k} .
\end{aligned}
$$

In each of these four cases we have $c\left(g_{1}, \ldots, g_{k}, \overline{\bar{o}}\right)(j)=\bar{o}$ for all but possibly two integers $j$. We always have

$$
c\left(g_{1}, \ldots, g_{k}, \overline{\bar{o}}\right)(i)=c\left(u_{1}, \ldots, u_{k}, \bar{o}\right)
$$

and, depending on the choice for $g_{1}, g_{2}$, also

$$
\left.\begin{array}{l}
c\left(d_{i}, d_{i-1}, c_{3}, \ldots, c_{k}, \overline{\bar{o}}\right)(i+t+2) \\
c\left(d_{i}, d_{i-t-2}, c_{3}, \ldots, c_{k}, \overline{\bar{o}}\right)(i+1) \\
c\left(d_{i-t-3}, d_{i-1}, c_{3}, \ldots, c_{k}, \overline{\bar{o}}\right)(i-1) \\
c\left(d_{i-t-3}, d_{i-t-2}, c_{3}, \ldots, c_{k}, \overline{\bar{o}}\right)(i-t-2)
\end{array}\right\}=c\left(u_{2}, u_{1}, u_{3} \ldots, u_{k}, \bar{o}\right)
$$

In Case 1 , by Lemmas 2.10 and 2.8 we have that $c\left(u_{2}, u_{1}, u_{3} \ldots, u_{k}, \bar{o}\right)=$ $-c\left(u_{1}, \ldots, u_{k}, \bar{o}\right)$. Hence $\sum_{j \in \mathbb{Z}} w(j)=\bar{o}$ and the result follows.

In Case 2, $a_{1}=a_{2}$ yields $c\left(u_{1}, \ldots, u_{k}, \bar{o}\right)=c\left(u_{2}, u_{1}, u_{3} \ldots, u_{k}, \bar{o}\right)$. Since the difference between $i$ and the second index at which $w$ is not $\bar{o}$ is always odd, we obtain $\sum_{j \in \mathbb{Z}}(-1)^{j} w(j)=\bar{o}$. Again the result follows.

Lemma 3.5. $g=(\ldots, \bar{o}, \underset{0}{e}, \bar{o}, \ldots)$ is not in $D$.

Proof. Immediately from Lemma 3.4.

We now verify that the first condition of Lemma 3.2 holds for $N:=2|B|(2|B|-1)$. Let $\varphi: \mathbf{D} \rightarrow \mathbf{B}$ be a homomorphism with $\operatorname{kernel} \operatorname{ker}(\varphi)=: \theta$. We will show that $\operatorname{ker}\left(\left.\varphi\right|_{D_{0}}\right)$ has only one block of size greater than $N$.

First we establish a bound on the number of elements $v_{i, i+1}$ that are not congruent to $\overline{\bar{O}}$ modulo $\theta$.

Lemma 3.6. Let $I:=\left\{i \in \mathbb{Z}: v_{i, i+1} \not_{\theta} \overline{\bar{o}}\right\}$. Then $|I| \leq 2|B|$.

Proof. Suppose that $|I|>2|B|$. Then there are $p, q, r \in I$ with $p<q<r$ such that $d_{p} \equiv_{\theta} d_{q} \equiv_{\theta} d_{r}$. Observe that

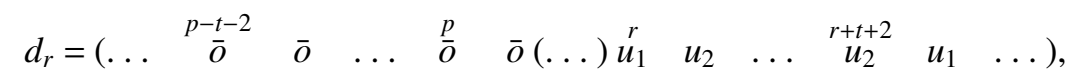

$$
\begin{aligned}
& d_{p-t-2}=\left(\begin{array}{lllllllllll}
\ldots & u_{1} & u_{2} & \ldots & u_{2} & u_{1}(\ldots) \bar{o} & \bar{o} & \ldots & \bar{o} & \bar{o} & \ldots
\end{array}\right) \text {, }
\end{aligned}
$$


where the (...) stands for arbitrarily many (potentially zero) terms $\bar{o}$. In particular,

$$
\forall i \in \mathbb{Z}, \quad d_{p-t-2}(i)=\bar{o} \text { or } d_{r}(i)=\bar{o} .
$$

Thus

$$
v_{p, p+1}=f\left(d_{p}, d_{p-t-2}, c_{3}, \ldots, c_{k}, \overline{\bar{o}}\right) \equiv_{\theta} f\left(d_{r}, d_{p-t-2}, c_{3}, \ldots, c_{k}, \overline{\bar{o}}\right)=\overline{\bar{o}}
$$

which contradicts $p \in I$. Hence $|I| \leq 2|B|$.

Next we show that if $L$ is a small enough integer and $J$ a long enough interval with $v_{l, l+1} \equiv_{\theta} \overline{\bar{o}}$ for all $l \in J$, then $v_{L, l} \equiv_{\theta} \overline{\bar{o}}$ for all $l \in J$.

Lemma 3.7. Let $L<\min (I)$, and let $J:=\{j, \ldots, j+2|B|-2\}$ such that $I \cap J=\emptyset$. Then $v_{L, l} \equiv_{\theta} \overline{\bar{o}}$ for all $l \in J$.

Proof. Choose an integer $n \geq 1$ such that $j+2|B|-1-L \leq(2 n+1)(t+3)$. For $i \in \mathbb{Z}$ define

$$
w_{i}:=m\left(\ldots\left(m\left(d_{i}, d_{i+(t+3)}, d_{i+2(t+3)}\right), \ldots\right), d_{i+(2 n-1)(t+3)}, d_{i+2 n(t+3)}\right) .
$$

Let $-u_{2}:=m\left(\bar{o}, u_{2}, \bar{o}\right)$. It is straightforward to see that

$$
w_{i}(l)= \begin{cases}u_{1} & \text { if } l \in\{i, i+(2 n+1)(t+3)\} \\ u_{2} & \text { if } l=i+1+\lambda(t+3) \text { for some } \lambda \in\{0,2, \ldots, 2 n\}, \\ u_{2} & \text { if } l=i+t+2+\lambda(t+3) \text { for some } \lambda \in\{0,2, \ldots, 2 n\}, \\ -u_{2} & \text { if } l=i+1+\lambda(t+3) \text { for some } \lambda \in\{1,3, \ldots, 2 n-1\}, \\ -u_{2} & \text { if } l=i+t+2+\lambda(t+3) \text { for some } \lambda \in\{1,3, \ldots, 2 n-1\}, \\ \bar{o} & \text { otherwise. }\end{cases}
$$

That is,

$$
\begin{gathered}
w_{i}=\left(\ldots, \bar{o}, u_{1}, u_{2}, \bar{o}, \ldots, \bar{o}, u_{2}, \underset{i+t+3}{\bar{o}},-u_{2}, \bar{o}, \ldots, \bar{o},-u_{2}, \underset{i+2(t+3)}{\bar{o}}, u_{2}, \bar{o},\right. \\
\left.\ldots, \bar{o},-u_{2}, \underset{i+2 n(t+3)}{\bar{o}}, u_{2}, \bar{o}, \ldots, \bar{o}, u_{2}, \underset{i+(2 n+1)(t+3)}{u_{1}}, \bar{o}, \ldots\right) .
\end{gathered}
$$

Let $T:=(2 n+1)(t+3)$.

Now consider $w_{i}$ and $w_{i-1}$ given by

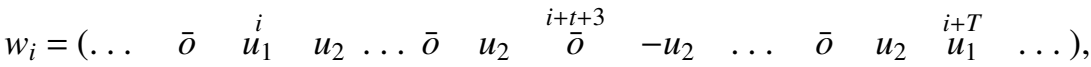

$$
\begin{aligned}
& w_{i-1}=\left(\begin{array}{llllllllllllll}
\ldots & u_{1} & u_{2} & \bar{o} & \ldots & u_{2} & \bar{o} & -u_{2} & \bar{o} & \ldots & u_{2} & u_{1} & \bar{o} & \ldots
\end{array}\right) \text {. }
\end{aligned}
$$

Then

$$
f\left(w_{i}, w_{i-1}, c_{3}, \ldots, c_{k}, \overline{\bar{o}}\right)=v_{i, i+T-1},
$$

where in Case 2 we used the fact that $T-1$ is odd, and hence $v_{i, i+T-1}(i+T-1)=+e=$ $f\left(u_{2}, u_{1}, c_{3}, \ldots, c_{k}, \bar{o}\right)$. 
We have integers $p, q, r$ with $j-T+1 \leq p<q<r \leq j-T+2|B|+1$ such that $w_{p} \equiv_{\theta} w_{q} \equiv_{\theta} w_{r}$. Since $p+1<r<p+t$, we have (similar to the argument for Lemma 3.6)

$$
\begin{array}{cccccccc}
w_{p-1}=(\ldots & u_{1} & u_{2} & \bar{o} & (\ldots) & \bar{o} & \bar{o} & (\ldots) \\
w_{r}=(\ldots & \bar{o} & \bar{o} & \bar{o} & (\ldots) & u_{1} & u_{2} & (\ldots) \\
& & p & \leq q & & r & \leq(p-1)+t+1 & \\
u_{2} & \bar{o} & -u_{2} & (\ldots) & \bar{o} & \bar{o} & \bar{o} & (\ldots) \\
\bar{o} & \bar{o} & \bar{o} & (\ldots) & u_{2} & \bar{o} & -u_{2} & (\ldots) \\
(p-1)+t+2 & & \leq q+t+2 & & r+t+2 & & \leq(p-1)+2(t+3)-2 & \\
& & & & & & & \\
u_{2} & u_{1} & (\ldots) & \bar{o} & \bar{o} & \ldots) & & \\
\bar{o} & \bar{o} & (\ldots) & u_{2} & u_{1} & \ldots) & & \\
(p-1)+T-1 & \leq q+T-1 & & & r+T & & &
\end{array}
$$

We observe that

$$
\forall i \in \mathbb{Z}, \quad w_{p-1}(i)=\bar{o} \text { or } w_{r}(i)=\bar{o} .
$$

Thus

$$
v_{p, p+T-1}=f\left(w_{p}, w_{p-1}, c_{3}, \ldots, c_{k}, \overline{\bar{o}}\right) \equiv_{\theta} f\left(w_{r}, w_{p-1}, c_{3}, \ldots, c_{k}, \overline{\bar{o}}\right)=\overline{\bar{o}} .
$$

Since $p \leq j-T+2|B|-1 \leq L$ and $v_{l, l+1} \equiv_{\theta} \overline{\bar{o}}$ for all $l \leq L$, this yields

$$
v_{L, p+T-1} \equiv_{\theta} \overline{\bar{o}} .
$$

Note that $p+T-1$ is in $J$. Since $v_{l, l+1} \equiv_{\theta} \overline{\bar{o}}$ for all $l \in J$, we finally obtain

$$
v_{L, l} \equiv_{\theta} \overline{\bar{o}} \quad \text { for all } l \in J .
$$

Lemma 3.8. Let $L$ be an even negative integer with $L<\min (I)$. Then

$$
\left|\left\{i \in \mathbb{N}: v_{0, i} \not_{\theta}-v_{L, 0}\right\}\right| \leq 2|B|(2|B|-1) .
$$

Proof. Let $i \in \mathbb{N}$. Note that

$$
v_{L, i}=v_{L, 0}+v_{0, i} .
$$

Assume that $v_{0, i} \equiv_{\theta}-v_{L, 0}$. Then $v_{L, i} \not_{\theta} \overline{\bar{o}}$. So, by Lemma 3.7, we have $j \in I$ such that $|i-j| \leq|B|-1$. Together with Lemma 3.6 this yields the result.

Finally, all assumptions of Lemma 3.2 are satisfied for $N=2|B|(2|B|-1)$ and $\mathbf{D}$ and $D_{0}$ as above. It follows that $\mathbf{B}$ is not dualizable. Theorem 3.1 is proved.

Proof of Theorem 1.1. Let $\mathbf{A}$ be a finite nonabelian nilpotent algebra of finite type that splits into a direct product of prime power algebras. Then $\mathbf{A}$ is supernilpotent by Theorem 2.4. Hence A satisfies the assumptions of Theorem 3.1 with $\alpha:=1$, and the result follows. 


\section{A clone theoretic condition for duality}

In the next section we will give an example of a dualizable algebra that limits how far Theorem 3.1 can be generalized. We will show duality by a novel approach using clone theory that was suggested by Willard [17] and further developed by Davey et al. [8]. We note that in [8] the authors develop an extension of the duality theory from [4]; see the appendix for a discussion of the clone theoretic approach in this new so-called symmetric setting and explanations about the (minor) differences between the theories. As explained in the appendix to [8], results stated in one setting can be readily translated to the corresponding results in the other setting.

In the present section we state Willard's clone theoretic condition that is sufficient for dualizability in Corollary 4.3. For the convenience of the reader, we give a proof based on well-known results from the book [4], namely the third duality theorem (Theorem 3.1.6) and the duality compactness theorem (Theorem 2.2.11); see the appendix for a development of the corresponding results in the symmetric setting of [8].

We briefly recall the definition of duality from [4]. Let $\mathbf{A}_{0}$ be a finite algebra with universe $A_{0}$, and consider a topological structure ${\underset{\sim}{\mathbf{A}}}_{1}:=\left\langle A_{0}, \mathcal{F}, \mathcal{R}, \tau\right\rangle$ on $A_{0}$, where $\mathcal{F}$ is a set of total or partial operations, $\mathcal{R}$ is a set of relations, and $\tau$ is the discrete topology, such that each operation of $\mathbf{A}$ preserves each relation $R \in \mathcal{R}$ and the graph of each $F \in \mathcal{F}$. Then ${\underset{\sim}{\mathbf{A}}}_{1}$ is called an alter ego of $\mathbf{A}_{0}$.

The aim of duality theory is to find suitable choices of $\mathcal{F}$ and $\mathcal{R}$ in order to set up a dual representation between two different categories. One corresponds to the quasivariety $\mathcal{A}:=\mathbb{I S P}\left(\mathbf{A}_{0}\right)$ generated by $\mathbf{A}_{0}$, consisting of all isomorphic copies of subalgebras of powers of $\mathbf{A}_{0}$. The other corresponds to the topological quasivariety $\mathcal{X}:=\mathbb{I} \mathbb{S}_{C} \mathbb{P}^{+}\left({\underset{\sim}{1}}_{1}\right)$ of isomorphic copies of closed substructures of powers of $\mathbf{\sim}_{1}$, where powers are taken over nonempty index sets. The morphisms of the categories are the homomorphisms and continuous homomorphisms, respectively, among their objects.

We can set up mappings $D: \mathcal{A} \rightarrow \mathcal{X}$ and $E: \mathcal{X} \rightarrow \mathcal{A}$. For $\mathbf{A} \in \mathcal{A}$ let $D(\mathbf{A})$ be the substructure of $\underset{\sim}{\mathbf{A}_{1}^{A}}$ whose universe consists of all homomorphisms from $\mathbf{A}$ to $\mathbf{A}_{0}$. For $\mathbf{X} \in \mathcal{X}$ let $E(\mathbf{X})$ be the subalgebra of $\mathbf{A}_{0}^{X}$ whose universe consists of all continuous homomorphisms from $\mathbf{X}$ to $\underset{\sim}{\mathbf{A}_{1}}$ (we remark in passing that $D$ and $E$ can be extended to contravariant functors between $\mathcal{A}$ and $\mathcal{X}$ ).

Now for each $\mathbf{A} \in \mathcal{A}$ we have a natural embedding $e_{\mathbf{A}}: \mathbf{A} \rightarrow E D(\mathbf{A})$ given by evaluation, that is, $e_{\mathbf{A}}(a)$ is given by $h \mapsto h(a)$ for each continuous homomorphism $h \in D(\mathbf{A})$.

We say that $\underset{\sim}{\mathbf{A}_{1}}$ dualizes $\mathbf{A}_{0}$ if $e_{A}$ is an isomorphism for each $\mathbf{A} \in \mathcal{A}$. $\mathbf{A}_{0}$ is dualizable if there is a structure $\underset{\sim}{\mathbf{A}_{1}}$ that dualizes $\mathbf{A}_{0}$. We say that $\underset{\sim}{\mathbf{A}_{1}}$ dualizes $\mathbf{A}_{0}$ at the finite level if $e_{A}$ is an isomorphism for each finite $\mathbf{A} \in \mathcal{A}$.

The next definitions will be used in providing a clone theoretic approach to dualizability. Let $\mathbf{A}$ be an algebra. A subset $D$ of some finite power $A^{k}$ of $A$ is conjunct-atomic definable (c.a.d.) [8] over $\mathbf{A}$ if it is definable by a conjunction of atomic formulas over $\mathbf{A}$. That is, $D$ is c.a.d. over $\mathbf{A}$ if there exist $f_{1}, \ldots, f_{l}, g_{1}, \ldots, g_{l} \in$ 
$\mathrm{Clo}_{k}(\mathbf{A})$ such that

$$
D=\left\{x \in A^{k}: f_{1}(x)=g_{1}(x), \ldots, f_{l}(x)=g_{l}(x)\right\} .
$$

Note that the empty set $\emptyset$ may be c.a.d. over A. In [4, page 66] c.a.d. relations are called term closed.

Let $R \subseteq A^{n}$, and for $D \subseteq A^{k}$ let $f$ be a partial operation $f: D \rightarrow A$. We can extend $f$ to a partial operation $f^{A^{n}}$ on $A^{n}$ by evaluating $f$ coordinatewise. We say that $f$ preserves $R$ if

$$
\forall r_{1}, \ldots, r_{k} \in R, \quad f^{A^{n}}\left(r_{1}, \ldots, r_{k}\right) \in R \text { whenever defined. }
$$

We denote the set of all restrictions of term operations on $\mathbf{A}$ to c.a.d. domains by $\mathrm{Clo}_{\text {cad }}(\mathbf{A})$. We say that the partial clone $\mathrm{Clo}_{\text {cad }}(\mathbf{A})$ is finitely related if there exists a finite set $\mathcal{R}=\left\{R_{1}, \ldots, R_{l}\right\}$ of subuniverses of finite powers of $\mathbf{A}$ such that the following are equivalent for every partial operation $f$ on $A$ with c.a.d. domain over $\mathbf{A}$ :

(1) $f$ preserves the relations $R_{1}, \ldots, R_{l}$;

(2) $f \in \mathrm{Clo}_{\text {cad }}(\mathbf{A})$.

We note that the implication $(2) \Rightarrow$ (1) is trivially satisfied as $R_{1}, \ldots, R_{l}$ are subuniverses of powers of $\mathbf{A}$.

We will need the following variants of well-known results.

Theorem 4.1 [4, Theorem 3.1.6]. Let $\mathcal{R}:=\left\{R_{1}, \ldots, R_{l}\right\}$ be a finite set of relations on $A$, the universe of the finite algebra $\mathbf{A}$, and let $\underset{\sim}{\mathbf{A}}:=\langle A, \emptyset, \mathcal{R}, \tau\rangle$ be with $\tau$ the discrete topology on A. The following are equivalent.

(1) The structured space $\mathbf{A}$ dualizes $\mathbf{A}$ at the finite level.

(2) For any $k \in \mathbb{N}$ and any substructure $\underset{\sim}{\mathbf{D}}$ of ${\underset{\sim}{\mathbf{A}}}^{k}$ whose universe $D$ is c.a.d. over $\mathbf{A}$, every morphism $\mathbf{\mathbf { D }} \rightarrow \underset{\sim}{\mathbf{A}}$ extends to a total $\hat{k}$-ary term function on $\mathbf{A}$.

(3) $\mathrm{Clo}_{\mathrm{cad}}(\mathbf{A})$ is finitely related by the relations $R_{1}, \ldots, R_{l}$.

Proof. (1) $\Leftrightarrow(2)$ is a special case of the finite level case of [4, Theorem 3.1.6]. For $(2) \Rightarrow$ (3) consider a partial operation $f: D \rightarrow A$ with domain $D \subseteq A^{k}$ c.a.d. over $\mathbf{A}$ such that $f$ preserves the relations in $\mathcal{R}$. Then $D$ induces a substructure $\underset{\tilde{\mathbf{D}}}{\mathbf{D}}$ of $\underset{\sim}{\mathbf{A}^{k}}$. As $D$ is finite and $\tau$ discrete, and $f$ preserves all relations $R$ in $\mathcal{R}, f$ is actually a morphism from $\underset{\sim}{\mathbf{D}}$ to $\underset{\sim}{\mathbf{A}}$ in the sense of [4, page 21]. By (2), $f$ extends to a total term operation on A, that is, $\tilde{f} \in \mathrm{Clo}_{\text {cad }}(\mathbf{A})$. Hence we have (3).

The converse implication (3) $\Rightarrow$ (2) follows similarly. Let $\underset{\sim}{\mathbf{D}}$ be a substructure of $\underset{\sim}{\mathbf{A}}{ }^{k}$ whose universe is c.a.d. over $\mathbf{A}$, and let $f: \underset{\sim}{\mathbf{D}} \rightarrow \underset{\sim}{\mathbf{A}}$ be a morphism. Then $f$ preserves the relations $R_{1}, \ldots, R_{l}$ and $f \in \mathrm{Clo}_{\mathrm{cad}}(\mathbf{A})$ by (3). Thus $f$ extends to a term operation on $\mathbf{A}$, and we have (2).

Theorem 4.2. [4, Theorem 2.2.11]; independently Willard [18], Zadori [19]. Let A be a finite algebra. If the structure $\underset{\sim}{\mathbf{A}}$ is of finite type and dualizes $\mathbf{A}$ at the finite level, then $\underset{\sim}{\mathbf{A}}$ dualizes $\mathbf{A}$. 
Combining the above results (equivalently, combining the version of [8, Lemma 4.1] for the so-called usual setting with [4, Theorem 2.2.11]), we immediately get the following corollary.

Corollary 4.3 (Willard [17]). Let $\mathbf{A}$ be a finite algebra. If $\mathrm{Clo}_{\mathrm{cad}}(\mathbf{A})$ is finitely related by relations $R_{1}, \ldots, R_{l}$, then $\mathbf{A}$ is dualized by the finitary structure $\underset{\sim}{\mathbf{A}}:=$ $\left\langle A, \emptyset,\left\{R_{1}, \ldots, R_{l}\right\}, \tau\right\rangle$ with $\tau$ the discrete topology on $A$.

\section{Nilpotent and dualizable}

We give an example that shows that in Theorem 3.1 the condition that $\mathbf{A}$ has a nonabelian, supernilpotent congruence cannot be removed. In particular, dualizable nonabelian nilpotent Mal'cev algebras exist.

Throughout the remainder of this section let

$$
\mathbf{A}:=\left\langle\mathbb{Z}_{4},+, 1,\left\{2 x_{1} \ldots x_{k}: k \in \mathbb{N}\right\}\right\rangle .
$$

We note that $\mathbf{A}$ is nilpotent with center $\equiv_{2}$ but not supernilpotent. Observe that every reduct of finite type of $\mathbf{A}$ with group operation is inherently nondualizable by Theorem 1.1. Still we have the following result.

Theorem 5.1. A $:=\left\langle\mathbb{Z}_{4},+, 1,\left\{2 x_{1} \ldots x_{k}: k \in \mathbb{N}\right\}\right\rangle$ is dualizable by the alter ego $\underset{\sim}{\mathbf{A}}:=$ $\langle A, \emptyset, \mathcal{R}, \tau\rangle$, where $\mathcal{R}$ is the set of all subuniverses of $\mathbf{A}^{4}$.

Before proving the theorem we describe the term operations on $\mathbf{A}$. For $k \in \mathbb{N}$ and $v \in \mathbb{Z}_{4}^{k}$, define

$$
c_{v}: \mathbb{Z}_{4}^{k} \rightarrow \mathbb{Z}_{4}, x \mapsto \begin{cases}2 & \text { if } x \in v+2 \mathbb{Z}_{4}^{k}, \\ 0 & \text { otherwise. }\end{cases}
$$

Lemma 5.2. Let $f: \mathbb{Z}_{4}^{k} \rightarrow \mathbb{Z}_{4}$ with $f(0, \ldots, 0)=0$. Then $f \in \mathrm{Clo}_{k}(\mathbf{A})$ if and only if there exist $\lambda_{1}, \ldots, \lambda_{k} \in \mathbb{Z}_{4}$, there exist $v_{1}, \ldots, v_{l} \in \mathbb{Z}_{4}^{k}$ such that for all $x \in \mathbb{Z}_{4}^{k}$,

$$
f(x)=\sum_{i=1}^{k} \lambda_{i} x_{i}+c_{v_{1}}(x)+\cdots+c_{v_{l}}(x) .
$$

Proof. Any $f \in \mathrm{Clo}_{k}(\mathbf{A})$ with $f(0, \ldots, 0)=0$ can be written as

$$
f(x)=\sum_{i=1}^{k} \lambda_{i} x_{i}+g(x),
$$

where $g(x)$ is a sum of terms $2 x_{j_{1}} \ldots x_{j_{n}}$ for some $n \in \mathbb{N}$ and $j_{1}, \ldots, j_{n} \in\{1, \ldots, k\}$. It follows that $g(x)=g(x+y)$ for all $y \in 2 \mathbb{Z}_{4}^{k}$. Pick a set $v_{i}$ of representatives for those residue classes of $\mathbb{Z}_{4}^{k} \bmod 2 \mathbb{Z}_{4}^{k}$ which are mapped to 2 by $g$. Expression (5.1) follows. A

Conversely, let $f$ be of the form (5.1). If $v_{i}=\left(w_{1}, \ldots, w_{k}\right)$, then for all $x_{1}, \ldots, x_{k} \in$

$$
c_{v_{i}}\left(x_{1}, \ldots, x_{k}\right)=2\left(1+x_{1}+w_{1}\right) \cdots\left(1+x_{k}+w_{k}\right) .
$$

Hence all $c_{v_{i}}$ are in $\mathrm{Clo}_{k}(\mathbf{A})$, and hence $f \in \mathrm{Clo}_{k}(\mathbf{A})$. 
Next we determine the c.a.d. relations over $\mathbf{A}$.

Lemma 5.3. Let $D \subseteq \mathbb{Z}_{4}^{k}$ with $(0, \ldots, 0) \in D$, and let $B:=2 \mathbb{Z}_{4}^{k}$. Then $D$ is c.a.d. over $\mathbf{A}$ if and only if there exists a subgroup $U$ of $\langle B,+\rangle$ and $v_{1}, \ldots, v_{l} \in \mathbb{Z}_{4}^{k}$ such that $v_{i}-v_{j} \notin B$ whenever $i \neq j, 2 v_{i} \in U$ for all $i$, and

$$
D=v_{1}+U \cup \cdots \cup v_{l}+U .
$$

Proof. Assume that $D$ is c.a.d. We have $f_{1}, \ldots, f_{n} \in \mathrm{Clo}_{k}(\mathbf{A})$ such that $D=\left\{x \in \mathbb{Z}_{4}^{k}\right.$ : $\left.f_{1}(x)=0, \ldots, f_{n}(x)=0\right\}$. By Lemma 5.2 we have a $k \times n$ matrix $H$ over $\mathbb{Z}_{4}$ and vectors $b_{w} \in 2 \mathbb{Z}_{4}^{n}$ for $w \in\{0,1\}^{k}$ such that

$$
D=\bigcup_{w \in\{0,1\}^{k}}\left\{x \in w+B: H \cdot x=b_{w}\right\} .
$$

Let $U:=\{x \in B: H \cdot x=0\}$. Since $(0, \ldots, 0) \in D$, we have $b_{(0, \ldots, 0)}=(0, \ldots, 0)$ and $D \cap B=U$.

Let $w \in\{0,1\}^{k}$ such that $D \cap w+B \neq \emptyset$, say $v \in D \cap w+B$. It follows that $D \cap w+$ $B=v+U$. Since $H \cdot v=b_{w} \in 2 \mathbb{Z}_{4}^{n}$, we have $H \cdot(2 v)=0$ and $2 v \in U$. Hence $D$ is as in (5.2).

The converse implication of the lemma is now straightforward.

Finally, we show that $\mathrm{Clo}_{\text {cad }}(\mathbf{A})$ is finitely related.

Lemma 5.4. $\mathrm{Clo}_{\text {cad }}(\mathbf{A})$ is the set of partial operations with c.a.d. domain over $\mathbf{A}$ that preserve all subuniverses of $\mathbf{A}^{4}$.

Proof. Let $D \subseteq \mathbb{Z}_{4}^{k}$ be c.a.d. over $\mathbf{A}$, and let $f: D \rightarrow \mathbb{Z}_{4}$ preserve all subuniverses of $\mathbf{A}^{4}$. We will show that $f$ is the restriction of a term operation on $\mathbf{A}$. Since all constants are term operations on $\mathbf{A}$, we may assume without loss of generality that $(0, \ldots, 0) \in D$ and $f(0, \ldots, 0)=0$.

Let $U:=D \cap 2 \mathbb{Z}_{4}^{k}$. By Lemma 5.3 we have $v_{1}, \ldots, v_{l} \in \mathbb{Z}_{4}^{k}$ such that $v_{i}-v_{j} \notin 2 \mathbb{Z}_{4}^{k}$ whenever $i \neq j, 2 v_{i} \in U$ for all $i$, and $D=v_{1}+U \cup \cdots \cup v_{l}+U$. Note that

$$
M:=\left\{\left(\begin{array}{cc}
a & b \\
a+2 c & b+2 c
\end{array}\right): a, b, c \in A\right\}
$$

is a subuniverse of $\mathbf{A}^{2 \times 2}$ and hence preserved by $f$. Thus for all $x \in D, u \in U$ with $x+u \in D$,

$$
\left(\begin{array}{cc}
f(0, \ldots, 0) & f(x) \\
f(u) & f(x+u)
\end{array}\right) \in M .
$$

Hence

$$
\forall x \in D, u \in U, \quad x+u \in D \Rightarrow f(x+u)=f(x)+f(u) .
$$

In particular, $\left.f\right|_{U}$ is a group homomorphism from $U$ to $\mathbb{Z}_{4}$. Hence we have $t \in$ $\mathrm{Clo}_{k}\left(\left\langle\mathbb{Z}_{4},+\right\rangle\right)$ such that $\left.f\right|_{U}=\left.t\right|_{U}$. For $x \in D$ define

$$
g(x):=f(x)-t(x) .
$$


Let $i \in\{1, \ldots, l\}$. From (5.3) it follows that

$$
\forall u \in U, \quad g\left(v_{i}+u\right)=g\left(v_{i}\right) .
$$

We claim that

$$
g\left(v_{i}\right) \in 2 \mathbb{Z}_{4}
$$

For the proof consider

$$
h_{i}: \mathbb{Z}_{4} \rightarrow \mathbb{Z}_{4}, \quad x \mapsto g\left(x v_{i}\right) .
$$

Note that $h_{i}$ is indeed a total unary operation on $\mathbb{Z}_{4}$ since $2 v_{i} \in U$ implies that $\mathbb{Z}_{4} v_{i} \subseteq D$. Since $\mathrm{Clo}_{1}(\mathbf{A})$ embeds into $\mathbf{A}^{4}$, we have that $g$ preserves $\mathrm{Clo}_{1}(\mathbf{A})$ and consequently $h_{i} \in \mathrm{Clo}_{1}(\mathbf{A})$. Now $h_{i}(0)=g(0)=0$ and $h_{i}(2)=g\left(2 v_{i}\right)=0$. As $h_{i}$ is a term operation on A, this implies either $h_{i}(x)=0$ for all $x \in \mathbb{Z}_{4}$ or $h_{i}(x)=2 x$ for all $x \in \mathbb{Z}_{4}$. In any case $h_{i}(1)=g\left(v_{i}\right)$ is in $2 \mathbb{Z}_{4}$, which proves (5.5).

From (5.4) and (5.5) we obtain that for all $x \in D$,

$$
g(x)=\sum\left\{c_{v_{i}}(x): g\left(v_{i}\right)=2, i \in\{1, \ldots, l\}\right\} .
$$

Hence $f$ is the restriction of the term operation $t+\sum\left\{c_{v_{i}}: g\left(v_{i}\right)=2, i \in\{1, \ldots, l\}\right\}$.

Proof of Theorem 5.1. Since $\mathrm{Clo}_{\text {cad }}(\mathbf{A})$ is finitely related by the elements of $\mathcal{R}$ by Lemma 5.4, $\underset{\sim}{\mathbf{A}}$ dualizes A by Corollary 4.3.

\section{Problems}

Pontryagin duality yields that finite abelian groups are dualizable. In general, abelian algebras in congruence modular varieties are polynomially equivalent to modules over rings, which (if finite) are dualizable based upon an unpublished result of Keith Kearnes and Ágnes Szendrei. However, to our knowledge the following is still open.

Problem 6.1. Is every finite abelian algebra in a congruence modular variety dualizable?

More generally we would also like a characterization of all nilpotent algebras that are dualizable. Is supernilpotence the only obstacle for dualizability? Can we prove some kind of converse to Theorem 3.1?

Problem 6.2. Are the following equivalent for every finite nilpotent algebra $\mathbf{A}$ ?

(1) A is dualizable.

(2) For every algebra $\mathbf{B}$ in the variety generated by $\mathbf{A}$, all supernilpotent congruences of $\mathbf{B}$ are abelian.

\section{Acknowledgements}

The authors would like to thank the referee and Brian Davey for their helpful suggestions, Maria João Gouveia and Luís Sequeira for their support in conducting this research and writing this paper, as well as Michael Kinyon for his suggestions regarding the literature. 


\section{Appendix A. The clone theoretic approach in the symmetric setting}

In [8], Davey et al. develop a new approach to dualities that extends the theory (on the algebraic side) to structures with partial operations and relations, and also places it in a slightly more restricted setting (termed the symmetric setting). An extension to the duality theory from [4] is discussed in an appendix and termed the usual setting. The differences between the various settings are restricted to trivial members of the categories involved; however, they do affect part of the argument from Section 4. We have therefore decided to base the arguments in that section on [4], and to include this appendix showing how to derive the same conclusions in the symmetric setting of [8].

We need to make slight adjustments to the definitions from Section 4. In order to state the result in the language of [8] we will formulate it for structures, although we will only use structures that are effectively algebras. We will also slightly change the definition of $\mathrm{Clo}_{\text {cad }}$ to $\mathrm{Clo}_{\text {cad }}^{*}$. This change is actually not necessary in formulating the result, but reflects the fact that the symmetric setting avoids partial operations with empty domains. Note that the modifier * is not from [8] but is used here to clearly distinguish the two definitions.

Duality in [8] is defined not for algebras but for base structures. A base structure is a finite nonempty structure $\langle A, \mathcal{F}, \mathcal{R}\rangle$ where $\mathcal{F}$ is a set of partial operations on $A$ and $\mathcal{R}$ a set of relations on $A$, where (in the symmetric setting) all functions are nonnullary and have nonempty domain, and all relations are nonempty.

For our purposes, let $\left\langle A_{0}, \mathcal{F}\right\rangle$ be an algebra without nullary operations. This modification is inconsequential, as we will also (see below) exclude the empty structure from our modified version of $\mathcal{X}$. Hence we may replace any constants by the corresponding unary function with constant image. We will identify $\mathbf{A}_{0}$ with the base structure $\left\langle A_{0}, \mathcal{F}, \emptyset\right\rangle$, keeping the name $\mathbf{A}_{0}$ for both. We will leave it to the reader to check that dualizability of the algebra $\mathbf{A}_{0}$, as defined in Section 4, corresponds to dualizability of the base structure $\mathbf{A}_{0}$ in the so called usual setting of [8].

Duality in the context of the symmetric setting is characterized by replacing the category $\mathcal{A}$ with a slightly more restricted version of itself. Let $\mathcal{A}^{*}$ be the category $\operatorname{ISP}^{+}\left(\mathbf{A}_{0}\right)$ of all base structures isomorphic to substructures of powers of $\mathbf{A}_{0}$ over nonempty index sets, together with their morphisms. So when considered as a class of algebras, $\mathcal{A}^{*}$ either will equal $\mathcal{A}$ or can be obtained from $\mathcal{A}$ by removing all oneelement algebras. Similarly, on the topological side we replace $\mathcal{X}$ with $\mathcal{X}^{*}$, which is obtained from $\mathcal{X}$ by removing the empty structure and its morphisms (if present). We define the functors $D$ and $E$ as before, and we get a notion of duality or finite level duality in the symmetric setting by restricting the corresponding definitions to structures from $\mathcal{A}^{*}$.

As before, we define a subset $D \subseteq A_{0}^{k}$ for $k \geq 1$ to be conjunct-atomic definable (c.a.d.) over $\mathbf{A}_{0}$ if it is definable by a conjunction of atomic formulas over $\mathbf{A}_{0}$. The set of all finitary nonempty relations that are c.a.d. over $\mathbf{A}_{0}$ is called $\operatorname{Def}_{\mathrm{ca}}(\mathbf{A})$. We denote the set of all restrictions of term operations on $\mathbf{A}$ to domains in $\operatorname{Def}_{\mathrm{ca}}(\mathbf{A})$ by $\mathrm{Clo}_{\text {cad }}^{*}(\mathbf{A})$ (note that in [8], the elements of $\mathrm{Clo}_{\text {cad }}^{*}(\mathbf{A})$ are called structural functions). 
Let $R \subseteq A_{0}^{n}$ be nonempty. For nonempty $D \subseteq A_{0}^{k}$ a partial operation $f: D \rightarrow A_{0}$ preserves $R$ if

$$
\forall r_{1}, \ldots, r_{k} \in R, \quad f^{A^{n}}\left(r_{1}, \ldots, r_{k}\right) \in R \text { whenever defined, }
$$

where $f^{A^{n}}$ is defined as in Section 4. We say that $\mathrm{Clo}_{\text {cad }}^{*}\left(\mathbf{A}_{0}\right)$ is finitely related if there exist finitely many subuniverses $R_{1}, \ldots, R_{l}$ of finite powers of $\mathbf{A}_{0}$ such that the following are equivalent for every partial operation $f$ on $A$ with nonempty c.a.d. domain over $\mathbf{A}_{0}$ :

(1) $f$ preserves the relations $R_{1}, \ldots, R_{l}$;

(2) $f \in \mathrm{Clo}_{\text {cad }}^{*}\left(\mathbf{A}_{0}\right)$.

We are now able to state a sufficient condition for dualizability in the sense of [8]. Instead of a direct derivation in this setting (in a similar fashion to Section 4 with the role of the third duality theorem replaced by [8, Theorem 4.1]), we will instead translate between the theories.

Corollary A.1. Let $\mathbf{A}_{0}$ be a finite algebra without nullary operations. Assume that $\mathrm{Clo}_{\text {cad }}^{*}\left(\mathbf{A}_{0}\right)$ is finitely related by $R_{1}, \ldots, R_{l}$. Then $\mathbf{A}_{0}$ is dualized in the symmetric setting of $[8]$ by $\left\langle A_{0}, \emptyset,\left\{R_{1}, \ldots, R_{l}\right\}, \tau\right\rangle$, where $\tau$ is the discrete topology.

Proof. As the empty operation is compatible with all relations, we have that $\mathrm{Clo}_{\text {cad }}\left(\mathbf{A}_{0}\right)$ is also finitely related by $R_{1}, \ldots, R_{l}$ and hence is dualized by $\left\langle A_{0}, \emptyset,\left\{R_{1}, \ldots, R_{l}\right\}, \tau\right\rangle$ in the usual setting by Corollary 4.3. By [8, Lemma A.4] we get a duality in the symmetric setting if we remove all empty operations from $\mathbf{A}_{0}$ and replace all nullary operations of $\left\langle A_{0}, \emptyset,\left\{R_{1}, \ldots, R_{l}\right\}, \tau\right\rangle$ with unary ones. However, in our case these changes preserve both the algebraic and the topological structure. The result follows.

\section{References}

[1] E. Aichinger and N. Mudrinski, 'Some applications of higher commutators in Mal'cev algebras', Algebra Universalis 63(4) (2010), 367-403.

[2] R. H. Bruck, 'Contributions to the theory of loops', Trans. Amer. Math. Soc. 60(2) (1946), 245354.

[3] A. Bulatov, 'On the number of finite Mal'cev algebras', in: Proc. Dresden Conference 2000 (AAA 60) and the Summer School 1999, Contr. Gen. Alg., 13 (2001), 41-54.

[4] D. M. Clark and B. A. Davey, Natural Dualities for the Working Algebraist, Cambridge Studies in Advanced Mathematics, 57 (Cambridge University Press, Cambridge, 1998).

[5] D. M. Clark, B. A. Davey and J. G. Pitkethly, 'The complexity of dualisability: three-element unary algebras', Int. J. Algebra Comput. 13(3) (2003), 361-391.

[6] D. M. Clark, P. M. Idziak, L. R. Sabourin, Cs. Szabó and Ross Willard, 'Natural dualities for quasivarieties generated by a finite commutative ring', Algebra Universalis 46(1-2) (2001), 285320; the Viktor Aleksandrovich Gorbunov memorial issue.

[7] B. A. Davey, L. Heindorf and R. McKenzie, 'Near unanimity: an obstacle to general duality theory', Algebra Universalis 33 (1995), 428-439.

[8] B. A. Davey, J. G. Pitkethly and R. Willard, 'The lattice of alter egos', Int. J. Algebra Comput. 22(1) (2012), 36.

[9] R. Freese and R. N. McKenzie, Commutator Theory for Congruence Modular Varieties, London Mathematical Society Lecture Note Series, 125 (Cambridge University Press, Cambridge, 1987). 
[10] K. A. Kearnes, 'Congruence modular varieties with small free spectra', Algebra Universalis 42(3) (1999), 165-181.

[11] P. Mayr, 'Mal'cev algebras with supernilpotent centralizers', Algebra Universalis 65(2) (2011), 193-211.

[12] R. N. McKenzie, G. F. McNulty and W. F. Taylor, Algebras, Lattices, Varieties, Vol. I (Wadsworth \& Brooks/Cole Advanced Books \& Software, Monterey, CA, 1987).

[13] M. H. Nickodemus, 'Natural dualities for finite groups with abelian Sylow subgroups'. ProQuest LLC, Ann Arbor, MI, 2007, PhD Thesis, University of Colorado at Boulder.

[14] R. Quackenbush and Cs. Szabó, 'Nilpotent groups are not dualizable', J. Aust. Math. Soc. 72(2) (2002), 173-179.

[15] Cs. Szabó, 'Finite nilpotent rings are not dualizable', Algebra Universalis 42(4) (1999), 293-298.

[16] A. Vesanen, 'On p-groups as loop groups', Arch. Math. (Basel) 61 (1993), 1-6.

[17] R. Willard, 'Four unsolved problems in congruence permutable varieties', Talk at the Conference on Order, Algebra, and Logics, Nashville, 2007.

[18] R. Willard, 'New tools for proofing dualizability', in: Dualities, Interpretability and Ordered Structures (eds. J. Vaz de Carvalho and I. Ferreirim) (Centro de Algebra da Universidade de Lisboa, 1999), 69-74.

[19] L. Zadori, 'Natural Duality via a finite set of relations', Bull. Aust. Math. Soc. 51 (1995), 469-478.

WOLFRAM BENTZ, Centro de Álgebra, Universidade de Lisboa, Av. Prof. Gama Pinto, 2, 1649-003 Lisboa, Portugal e-mail: wfbentz@fc.ul.pt

PETER MAYR, Centro de Álgebra, Universidade de Lisboa, Av. Prof. Gama Pinto, 2, 1649-003 Lisboa, Portugal

and

Institute for Algebra, Johannes Kepler University Linz, Altenberger Straße 69, 4040 Linz, Austria

e-mail: pxmayr@gmail.com 